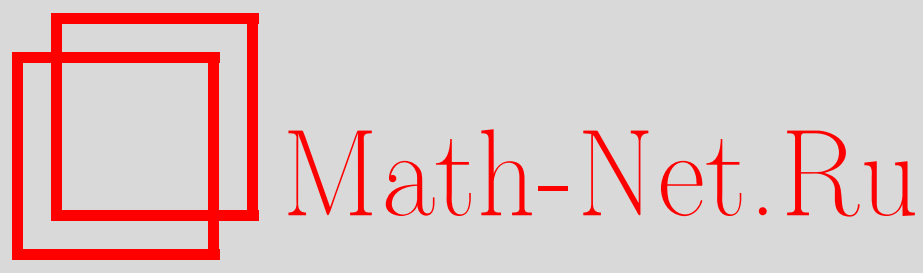

П. П. Кулиш, В. Д. Ляховский, О. В. Постнова, Функция кратностей для тензорных степеней модулей алгебры $A_{n}, T M \Phi, 2012$, том 171, номер 2, 283-293

DOI: https://doi.org/10.4213/tmf8371

Использование Общероссийского математического портала Math-Net.Ru подразумевает, что вы прочитали и согласны с пользовательским соглашением http://www.mathnet.ru/rus/agreement

Параметры загрузки:

IP : 3.89 .185 .249

26 апреля 2023 г., 13:25:13

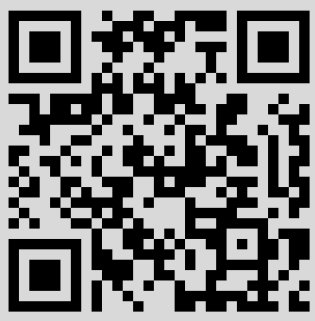




\title{
ФУНКЦИЯ КРАТНОСТЕЙ ДЛЯ ТЕНЗОРНЫХ СТЕПЕНЕЙ МОДУЛЕЙ АЛГЕБРЫ $A_{n}$
}

\begin{abstract}
Рассмотрено разложение $p$-й тензорной степени модуля $L^{\omega_{1}}$ алгебры $A_{n}$ на неприводимые модули: $\left(L^{\omega_{1}}\right)^{\otimes p}=\sum_{\nu} m(\nu, p) L^{\nu}$. Такая задача возникает, например, при нахождении спектра инвариантного гамильтониана спиновой цепочки с $p$ узлами. Для решения задачи предложено использовать свойства симметрии Вейля. Разработан алгоритм построения коэффициентов $m(\nu, p)$ как функций от $p$, который можно применять для степеней произвольного модуля. Выписано явное выражение для кратностей $m(\nu, p)$ в разложении степеней первого фундаментального модуля алгебры $s l(n+1)$. На основе полученных результатов найдены новые свойства систем ортогональных полиномов (мультивариантных полиномов Чебышёва). Возможно применение разработанного алгоритма к тензорным степеням модулей других простых алгебр Ли.
\end{abstract}

Ключевые слова: представления алгебр Ли, тензорные степени модулей, правила ветвления.

\section{1. ВВЕДЕНИЕ}

Задача нахождения коэффициентов слияния возникает в различных областях математической физики: в теории интегрируемых систем, конформной теории поля, квантовой гравитации и др. Мы изучаем свойства коэффициентов слияния с целью их применения к задачам теории интегрируемых систем [1]. Гамильтониан $\mathcal{H}$ интегрируемой спиновой цепочки с $p$ узлами задается на тензорном произведении $\bigotimes^{p} \mathbb{C}^{n}$ пространств состояний в узлах цепочки. Если гамильтониан инвариантен по отношению к действию алгебры Ли (или квантовой группы) $\mathfrak{g}$, т. е. $[\mathcal{H}, a]=0$ для $a \in \mathfrak{g}$, то его спектр имеет мультиплетную структуру и связан с разложением пространства $H$ состояний системы в прямую сумму пространств неприводимых представлений: $H=\bigoplus_{\nu} L^{\nu} \otimes \mathbb{C}^{m(\nu)}$, где $m(\nu)$ - кратности неприводимых модулей $L^{\nu}$.

Существуют различные методы нахождения коэффициентов слияния [2]. Наиболее распространенными среди них являются комбинаторные [3]-[6] и квантово-групповые [7]-[9]. Они могут использоваться в широкой области задач квантовой теории

${ }^{*}$ Санкт-Петербургское отделение Математического института им. В. А. Стеклова РАН, Санкт-Петербург, Россия. E-mail: kulish@pdmi.ras.ru

${ }^{\dagger}$ Санкт-Петербургский государственный университет, Санкт-Петербург, Россия. E-mail: lyakh1507@nm.ru, olgapostnova@mail.ru 
поля, но их применение для рассматриваемой нами спектральной задачи не дает желаемого результата: можно получить значения кратностей для конкретных (чаще всего простейших) примеров, но нахождение зависимости $m(\nu)$ от $p$ представляет значительную трудность.

Наша задача состоит в установлении явной зависимости коэффициентов слияния от степени $p$, что позволит исследовать асимптотические свойства спектра гамильтониана. Для этого необходим алгоритм, который:

1) основан на использовании симметрии Вейля (дабы избежать необходимости подсчета путей);

2) позволяет получить явное выражение для коэффициентов разложения на неприводимые представления для тензорных степеней фундаментальных представлений наименьшей размерности ("порождающих" представлений) алгебры $A_{n}$;

3) дает возможность выписать систему конечноразностных уравнений, с помощью которой можно рекуррентно получить выражение для коэффициентов $m(\nu, p)$ в случае произвольных $A_{n}$-модулей;

4) допускает распространение на другие алгебры Ли.

\section{2. ПОСТАНОВКА ЗАДАЧИ И ПРЕДВАРИТЕЛЬНЫЕ ЗАМЕЧАНИЯ}

Объектом нашего исследования являются тензорные степени первого фундаментального представления простой алгебры Ли серии $A_{n}(n-$ ранг алгебры). Базисные корни в $e$-базисе суть $\alpha_{1}=e_{1}-e_{2}, \ldots, \alpha_{n}=e_{n}-e_{n+1}$. Первый фундаментальный вес есть $\omega_{1}=e_{1}$, а первый фундаментальный модуль есть $L^{\omega_{1}}$. Рассмотрим тензорные степени $\left.\left(L^{\omega_{1}}\right)^{\otimes p}\right|_{p \in \mathbb{Z}_{+}}$и их разложение на неприводимые модули:

$$
\left(L^{\omega_{1}}\right)^{\otimes p}=\sum_{\nu} m(\nu, p) L^{\nu}
$$

Задача заключается в нахождении явной зависимости кратности $m(\nu, p)$ от $\nu$ и $p$.

Существуют различные методы нахождения кратностей подпредставлений в разложении тензорного произведения, однако установление с их помощью явной зависимости $m(\nu)$ от $p$ представляет значительные, подчас непреодолимые трудности. Для решения поставленной задачи мы используем формализм сингулярных элементов [10], основываясь на следующих соображениях. Рассмотрим кратность $m(\nu, p)$ модуля $L^{\nu}$ в разложении (1) как кратность его старшего веса в весовой диаграмме $N\left(\left(L^{\omega_{1}}\right)^{\otimes p}\right)$. Здесь функция $m(\nu, p)$ задана на множестве старших весов (множестве узлов решетки весов, принадлежащих замыканию главной камеры Вейля $\left.\overline{C^{(0)}}\right)$, и симметрия Вейля при таком подходе не учитывается. Построим естественное продолжение функции $m(\nu, p)$ на другие камеры Вейля. Если задать это продолжение как вейль-антиинвариантную функцию

$$
M(\mu, p)=\left\{\begin{array}{lll}
m(\nu, p) & \text { при } & \mu=\nu \in \overline{C^{(0)}}, \\
\operatorname{det}(w) m(\nu, p) & \text { при } & \mu=w(\nu+\rho)-\rho \notin \overline{C^{(0)}}
\end{array}\right.
$$

то функция $M(\mu, p)$ описывает кратности весов сингулярного элемента модуля $L^{\nu}$. Умножив обе части разложения тензорного произведения формальных характеров 
$\left(\mathrm{Ch}\left(L^{\omega_{1}}\right)\right)^{p}=\sum_{\nu} m(\nu, p) \mathrm{Ch} L^{\nu}$ на знаменатель Вейля, получим выражение, содержащее сингулярные элементы $\Psi^{L^{\nu}}$ :

$$
\Psi^{\left(\left(L^{\omega_{1}}\right)^{\otimes p}\right)}=\sum_{\nu} m(\nu, p) \Psi^{L^{\nu}}=\sum_{\xi \in P} M(\xi, p) e^{\xi} .
$$

В формальной алгебре $\varepsilon\left(A_{n}\right)$ сингулярный элемент модуля $L^{\mu}$ со старшим весом $\mu$ имеет вид

$$
\Psi^{L^{\mu}}=\sum_{w \in W} \operatorname{det}(w) e^{w(\mu+\rho)-\rho}
$$

где суммирование производится по группе Вейля $W$. Веса $w(\mu+\rho)-\rho$ можно назвать сингулярными весами модуля $L^{\mu}$. Областью определения функции $M(\mu, p)$ является весовое пространство $P$ в целом. Искомая функция $m(\mu, p)$ есть сужение $\left.M(\mu, p)\right|_{\overline{C^{(0)}}}$. В такой формулировке мы можем использовать свойства симметрии функции $M(\mu, p)$ для нахождения ее явного вида.

Помимо свойств симметрии, будем руководствоваться функциональными свойствами коэффициентов кратности, а именно полиномиальной зависимостью $M(\mu, p)$ от $p$. Последнее можно обосновать следующим образом. Рассмотрим $p$-кратное произведение модулей алгебры g. Задача разложения его на неприводимые модули эквивалентна задаче редукции неприводимого модуля $\left(L^{\mu}\right)^{\otimes p}$ алгебры $\bigoplus^{p} \mathfrak{g}$ до диагонально вложенной подалгебры g. Применим формализм сингулярных элементов. Проекция сингулярного элемента исходного модуля на подалгебру равна результату действия оператора $\Gamma_{A_{n}^{\text {diag }} \mapsto \bigoplus^{p} A_{n}}$ (так называемого веера вложения) на множество сингулярных весов модулей в разложении [11]. Применение этого оператора осложняется громоздкостью конструкции веера вложения для $\mathfrak{g}_{\mathrm{diag}} \mapsto \bigoplus^{p} \mathfrak{g}$ и сложностью сингулярного элемента. Однако после проведения соответствующих преобразований окончательное выражение для функции $M(\mu, p)$ самым существенным образом упрощается. Функция $M(\mu, p)$ оказывается полиномом по переменной $p$, степень которого растет по мере удаления от старшего веса $p \omega_{1}$.

Простота и симметричность функции $M(\mu, p)$ для конкретных значений весов $\mu$ наводит на мысль о существовании связи между значениями сингулярных кратностей при разных $p$. Необходимо записать эту связь как систему уравнений и выяснить возможность ее решения в явном виде.

\section{3. КОНЕЧНОРАЗНОСТНЫЕ УРАВНЕНИЯ}

3.1. Алгебра $s l(2)$. Чтобы понять, как в данной задаче возникают конечноразностные уравнения, рассмотрим простейший пример $\mathfrak{g}=s l(2)$. Для разложения степеней фундаментального представления алгебры $s l(2)$ может быть использован веер вложения (см. раздел 2). В частности, с его помощью можно построить рекуррентные соотношения и получить набор функций кратности, начиная с единицы кратности старшего веса (см. рис. 1).

Напомним, что кратности $m(\nu, p)$ в разложении (1) можно также получить с помощью подсчета путей на диаграмме Браттели (см. рис. 2). Нетрудно заметить, что полиномы на рис. 1 выражают зависимость числа путей на диаграмме от $p$. Диаграмма Браттели демонстрирует важную зависимость: кратность $m(\nu, p)$ в данной 


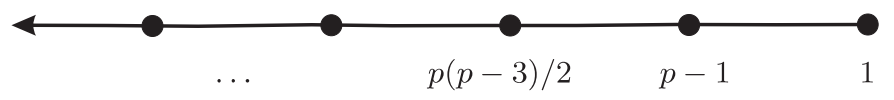

Рис. 1. Множество $\left\{m(\nu, p) \mid \nu \in C^{(0)}\right\}$ для степеней фундаментального модуля алгебры $s l(2)$. Старший вес имеет кратность 1 , слева от него веса, полученные сдвигом на корень $-\alpha$, поскольку в разложение тензорной степени входят модули одного класса конгруэнтности.

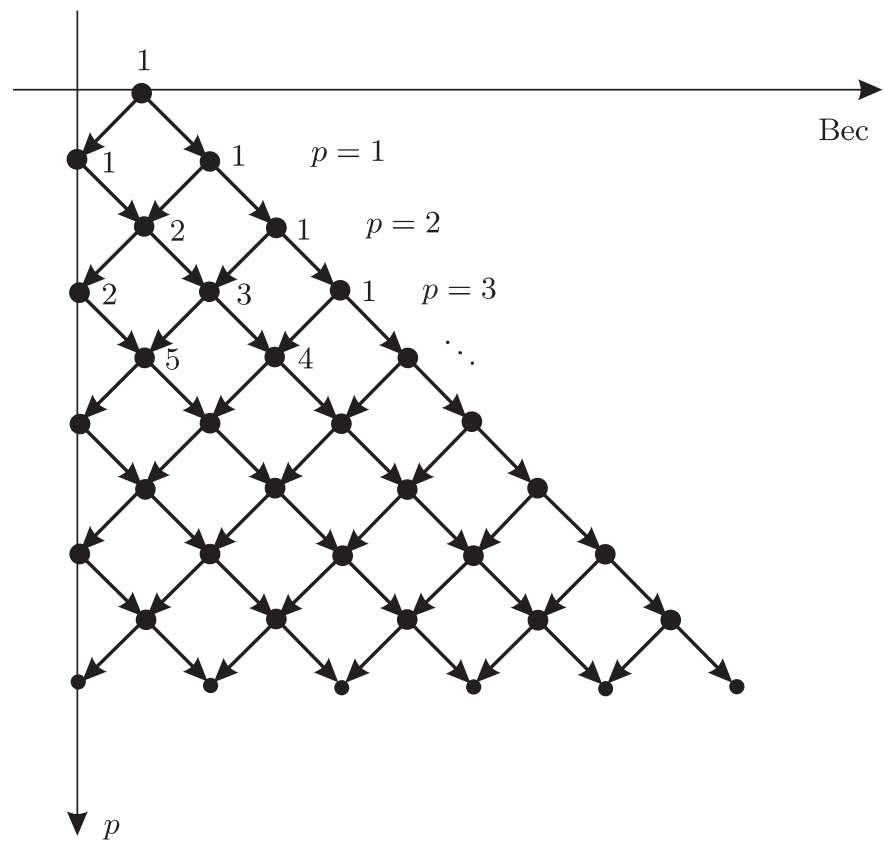

Рис. 2. Пути на диаграмме Браттели. По горизонтали отложены координаты старших весов подмодулей, по вертикали - тензорная степень исходного модуля. В узлах диаграммы указано число путей, которые ведут в данную точку из фундаментального веса, соответствующего $p=1$.

точке связана с кратностями для предыдущих значений $p$ соотношением

$$
m(\nu, p)=m(\nu-\omega, p-1)+m(\nu-\omega+\alpha, p-1),
$$

т. е. два пути ведут в данную точку из предыдущего ряда. Иными словами, кратности $m_{s l(2)}(\nu, p)$ подчинены конечноразностному уравнению $(2)$. Нетрудно проверить, что полиномы на рис. 1 удовлетворяют этому уравнению. Задача построения функции кратностей сводится к его решению.

3.2. Конечноразностные уравнения для алгебры $A_{n}$. Выше мы продемонстрировали на простейшем примере, как возникают конечноразностные уравнения для функции кратностей модулей алгебры $s l(2)$. Рассмотрим формирование конечноразностных уравнений в общем случае. Редукция $\mathfrak{g} \oplus \mathfrak{g} \downarrow \mathfrak{g}_{\text {diag }}$ прямой суммы до диагональной подалгебры индуцирует разложение формального характера модуля 
$L^{\mu} \otimes L^{\nu}$ алгебры $\mathfrak{g} \oplus \mathfrak{g}$ на подмодули диагональной подалгебры:

$$
\operatorname{Ch}\left(L^{\mu}\right) \operatorname{Ch}\left(L^{\nu}\right)_{\downarrow P_{\text {diag }}}=\sum_{\xi \in P_{\text {diag }}} m_{\xi}^{\mu \nu} \operatorname{Ch}\left(L^{\xi}\right),
$$

а в терминах сингулярных элементов

$$
\left(\frac{\Psi^{(\mu)} \Psi^{(\nu)}}{\Psi^{(0)} \Psi^{(0)}}\right)_{\downarrow P_{\text {diag }}}=\sum_{\xi \in P_{\text {diag }}} m_{\xi}^{\mu \nu} \frac{\Psi_{\text {diag }}^{(\xi)}}{\Psi_{\text {diag }}^{(0)}} .
$$

Используя коммутативность процедур произведения и проецирования

$$
\left(\Psi^{(\mu)} \Psi^{(\nu)}\right)_{\downarrow P_{\text {diag }}}=\Psi_{\downarrow P_{\text {diag }}}^{(\mu)} \Psi_{\downarrow P_{\text {diag }}}^{(\nu)}=\Psi_{\text {diag }}^{(\mu)} \Psi_{\text {diag }}^{(\nu)},
$$

получаем

$$
\left(\Psi_{\text {diag }}^{(0)}\right)^{-1} \Psi_{\text {diag }}^{(\mu)} \Psi_{\text {diag }}^{(\nu)}=\sum_{\xi \in P_{\text {diag }}} m_{\xi}^{\mu \nu} \Psi_{\text {diag }}^{(\xi)} .
$$

При этом элемент $\left(\Psi_{\text {diag }}^{(0)}\right)^{-1}$ можно отнести к любому из сомножителей $\Psi_{\text {diag }}^{(\mu)} \Psi_{\text {diag }}^{(\nu)}$, и умножение на этот элемент эквивалентно формированию диаграммы $N(L)$ модуля $L$ : имеем $\left(\Psi_{\text {diag }}^{(0)}\right)^{-1}: \Psi_{\text {diag }}^{(\mu)} \rightarrow N\left(L_{\text {diag }}^{\mu}\right)$. Таким образом,

$$
\sum_{\xi \in P_{\text {diag }}} m_{\xi}^{\mu \nu} \Psi_{\text {diag }}^{(\xi)}=N\left(L_{\text {diag }}^{(\mu)}\right) \Psi_{\text {diag }}^{(\nu)}=\Psi_{\text {diag }}^{(\mu)} N\left(L_{\text {diag }}^{(\nu)}\right)=\sum_{\xi \in P_{\text {diag }}} M_{\xi}^{\mu \nu} e^{\xi} .
$$

Рассмотрим старшие веса $\xi \in \overline{C^{(0)}}$. Значения, которые принимает функция $M_{\xi \in \overline{C^{(0)}}}^{\mu \nu}$, являются искомыми кратностями $m_{\xi}^{\mu \nu}$ модуля $L^{\xi}$ (одним из следствий данного соотношения является правило нахождения коэффициентов слияния, сформулированное в монографии [12]). Из формулы (3) следует, что значения функции кратностей определяются кратностями сингулярных весов, вклад веса зависит от значения детерминанта соответствующего преобразования группы Вейля.

Положим теперь $\mu=\omega, \nu=(p-1) \omega$, тогда

$$
\sum_{\xi \in P} M_{\xi}^{\bigotimes^{p} \omega} e^{\xi}=N\left(L^{\omega}\right) \Psi^{\left(\bigotimes^{p-1} \omega\right)}
$$

Будем рассматривать последнее равенство как рекуррентное уравнение для сингулярных элементов или, что эквивалентно, для коэффициентов $M_{\xi}^{\mu \nu}$ разложения сингулярного элемента в базисе формальных экспонент. Аналогично для случая $\mathfrak{g}=A_{n}$ и $\omega=\omega_{1}$ получаем систему конечноразностных уравнений

$$
M_{\xi}^{\bigotimes^{p} \omega}-M_{\xi-\omega}^{\bigotimes^{p-1} \omega}=M_{\xi-\omega+\alpha_{1}}^{\bigotimes^{p-1} \omega}+M_{\xi-\omega+\alpha_{1}+\alpha_{2}}^{\bigotimes^{p-1} \omega}+\cdots+M_{\xi-\omega+\alpha_{1}+\alpha_{2}+\cdots+\alpha_{n}}^{\bigotimes^{p-1} \omega} .
$$

3.3. Построение решений конечноразностных уравнений. Систему уравнений (5) вряд ли можно считать более простой, чем, например, комбинаторные правила подсчета путей. Выражение для функции $M_{\nu}^{\bigotimes^{p} \omega}$ можно получить рекуррентно для фиксированного $\nu$, но неясно, как искать общее решение.

Центральное утверждение настоящей работы состоит в следующем: решения системы конечноразностных уравнений (5) однозначно определяются симметрией 
Вейля, ограничениями области существования сингулярных элементов, граничными условиями и полиномиальной гладкостью функции кратности. Последнее свойство следует из рекуррентного соотношения, справедливого для функции кратности и индуцируемого веером вложений. Иными словами, мы будем искать решения $M_{a}^{\bigotimes^{p} \omega}$, удовлетворяющие следующим условиям.

1. Функции кратностей вне орбиты старшего веса $\mu=p \omega$ равны нулю. Это простое свойство для полиномиально гладкой функции требует уточнения: мы будем считать что все нули простые (вследствие рекуррентных соотношений), и требовать прежде всего, чтобы $M^{\bigotimes^{p} \omega}(\beta, p)$ обращалась в ноль вне орбиты старшего веса в узлах решетки доминантных весов. Правильное поведение в иных камерах Вейля будет обеспечено путем наложения условия вейль-антиинвариантности. Искомое решение удобно писать в координатах, согласованных с действием группы Вейля, где центр антиинвариантности отличается от нуля решетки $P$ на вектор $-\rho$.

2. Решение равно нулю на границах камер Вейля (сдвинутых на $-\rho$ ). Это является тривиальным свойством сингулярного элемента для модулей старшего веса конечномерной алгебры g. Как и в предыдущем случае, достаточно потребовать обращения решения в нуль на границах главной камеры. Простейшей функцией, обеспечивающей требуемое поведение, является произведение координат веса (в базисе фундаментальных весов).

3. Решение антиинвариантно по отношению к преобразованиям Вейля ( $W$-антиинвариантность): для $w \in W$

$$
w \circ M^{\bigotimes^{p} \omega}(\beta, p)=\varepsilon(w) M^{\bigotimes^{p} \omega}(\beta, p) .
$$

Поскольку при построении $W$-антиинварианта нули искомой функции необходимо сохранить, решение представляется в виде произведения элементов $W$-орбиты выражения, полученного на предыдущих этапах.

4. Искомое решение фиксируется граничным условием $M^{\otimes^{p} \omega}(p \omega+\rho, p)=1$.

В справедливости полученного выражения для решения можно удостовериться, подставив его в рекуррентные уравнения (5).

3.4. Решение конечноразностных уравнений на примере алгебры $A_{2}$. Пусть $\nu$ - доминантный вес алгебры $A_{2}$. Введем параметры $a_{1}, a_{2}$, определяемые равенством $\nu+\rho=a_{1} \omega_{1}+a_{2} \omega_{2}$. В случае алгебры $A_{2}$ для расширенной области определения функции кратности достаточно использовать три камеры Вейля (см. рис. 3). Старшие веса задаются координатами $a_{1}, a_{2}$ в базисе $\left\{\widetilde{\omega}_{1}, \widetilde{\omega}_{2}\right\}$, где начало отсчета сдвинуто на $-\rho$. Рассмотрим множество сингулярных весов для модуля $L^{\bigotimes^{p} \omega}$. Условия 1-4 определяют структуру функции $M^{\bigotimes^{p} \omega}$ следующим образом.

1. Условию 1 соответствует множитель (см. рис. 3)

$$
\frac{1}{\Gamma\left(\left(p-\left(a_{1}+2 a_{2}\right)+6\right) / 3\right)},
$$

который гарантирует, что все точки справа от границы множества аномальных весов имеют кратность 0. С ростом $p$ эта граница уходит вправо (из рекуррентных соотношений видно, что каждый такой множитель имеет кратность 1).

2. Требование равенства нулю функции кратности на сдвинутых границах камер Вейля позволяет определить множители $a_{1}$ и $a_{2}$. Первый из них приводит к обращению в ноль функции в узлах с координатами $r_{1} \widetilde{\omega}_{2}$, второй - в узлах с координатами $r_{2} \widetilde{\omega}_{1}$. 


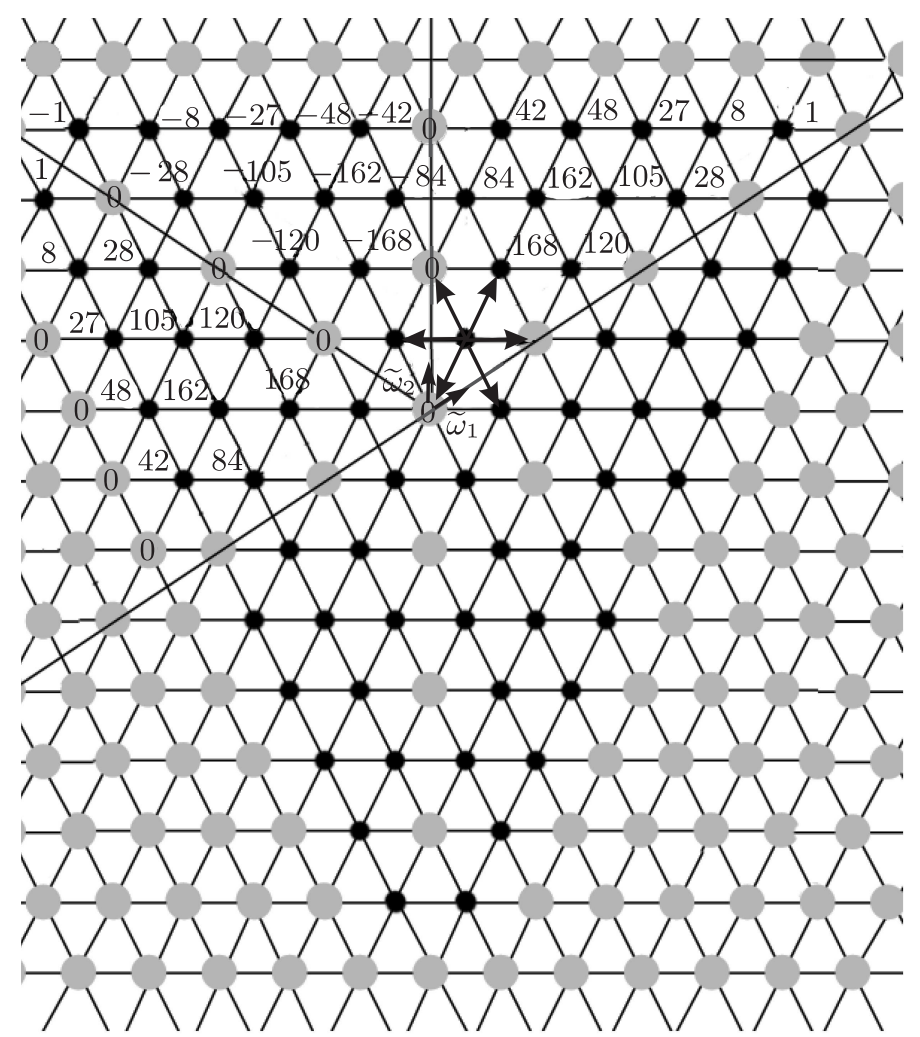

Рис. 3. Алгебра $A_{2}$, множество сингулярных весов для тензорной степени $p=9$. Черными точками обозначены веса с ненулевыми аномальными кратностями. Старший вес имеет кратность 1. Серые точки имеют кратность 0. Область определения функции кратности состоит из трех камер Вейля.

3. Чтобы удовлетворить требование антиинвариантности, рассмотрим действие группы Вейля на вес,

$$
s_{\alpha_{1}}\left(a_{1} \widetilde{\omega}_{1}+a_{2} \widetilde{\omega}_{2}\right)=-a_{1} \widetilde{\omega}_{1}+\left(a_{1}+a_{2}\right) \widetilde{\omega}_{2}, \quad s_{\alpha_{2}}\left(a_{1} \widetilde{\omega}_{1}+a_{2} \widetilde{\omega}_{2}\right)=\left(a_{1}+a_{2}\right) \widetilde{\omega}_{1}-a_{2} \widetilde{\omega}_{2},
$$

и построим простейшие антиинвариантные комбинации множителей, найденных в пп. 1,2 :

$$
\frac{1}{\Gamma\left(\left(p-\left(a_{1}+2 a_{2}\right)+6\right) / 3\right)} \frac{1}{\Gamma\left(\left(p+\left(2 a_{1}+a_{2}\right)+6\right) / 3\right)} \frac{1}{\Gamma\left(\left(p+\left(-a_{1}+a_{2}\right)+6\right) / 3\right)}
$$
и $a_{1} a_{2}\left(a_{1}+a_{2}\right)$.

4. Условие $M_{p \omega}^{\bigotimes^{p} \omega}=1$ окончательно фиксирует искомое выражение:

$$
\frac{\Gamma(p+1) a_{1} a_{2}\left(a_{1}+a_{2}\right)}{\Gamma\left(\left(p-\left(a_{1}+2 a_{2}\right)+6\right) / 3\right) \Gamma\left(\left(p+\left(2 a_{1}+a_{2}\right)+6\right) / 3\right) \Gamma\left(\left(p+\left(-a_{1}+a_{2}\right)+6\right) / 3\right)} .
$$


Можно непосредственно убедиться в том, что полученная функция является решением системы конечноразностных уравнений (5) и, следовательно, дает явный вид функции $M^{\otimes^{p} \omega}$.

3.5. Решение конечноразностных уравнений в случае алгебры $A_{n}$. Предложенный выше алгоритм допускает обобщение, справедливое для всякой алгебры $A_{n}$. Для $p$-й тензорной степени первого фундаментального модуля получим следующее выражение для кратностей неприводимых подмодулей:

$$
\begin{aligned}
& M_{\left(A_{n}\right)\left\{a_{i}\right\}}^{\otimes^{p} \omega}=p ! \prod_{i=1}^{n} a_{i} \prod_{j=1}^{n-1}\left(a_{j}+a_{j+1}\right) \ldots \prod_{j=1}^{2}\left(\sum_{k=1}^{n-1} a_{j+k}\right)\left(\sum_{k=1}^{n} a_{k}\right) \times \\
& \quad \times\left\{\prod_{l=0}^{n}\left(\frac{1}{n+1}\left(p+\frac{n(n+1)}{2}+\sum_{s=1}^{l}\left(-s a_{s}\right)+\sum_{t=l}^{n-1}(n-t) a_{t+1}\right)\right) !\right\}^{-1} .
\end{aligned}
$$

Проверить справедливость полученной формулы можно непосредственно, подставив данное выражение в систему уравнений (5).

Из вида функции $M_{\left(A_{n}\right)\left\{a_{i}\right\}}^{\bigotimes^{p} \omega}$ вытекают следующие важные свойства кратностей неприводимых компонент разложения.

1. Существуют "протыкающие" полиномы. Из полученной формулы видно, что одинаковую зависимость от $p$ (один и тот же полином) имеют разные старшие веса при разных $p$. Такие полиномы “протыкают" плоскости постоянства $p$ в разных точках, связанных линейными соотношениями.

2. Кратности весов, находящихся на внешней границе главной камеры Вейля, для алгебры $s l(m)$ совпадают с кратностями весов в главной камере Вейля для алгебры $s l(m-1)$. Это свойство обусловлено структурой фундаментального модуля рассматриваемых алгебр.

\section{6. Функция кратностей для степеней произвольного представления} алгебры $A_{1}$. Для алгебры $A_{1}$ произвольный неприводимый модуль $L^{[n]}$ старшего веса $\mu=n \omega$ (здесь удобнее использовать вместо старшего веса $\mu$ его индекс Дынкина $[n])$ можно выразить через комбинации степеней фундаментального модуля $L^{\omega}$. Используя этот факт, получаем общую формулу для кратности подмодуля $L^{\mu}$ со старшим весом $\mu=(a-1) \omega$ в $p$-й тензорной степени модуля $L_{s l(2)}^{[n]}$ :

$$
\begin{aligned}
M_{a}^{[n] \otimes^{p}=} & \sum_{k_{0}+k_{1}+\cdots+k_{[n / 2]}=p}(-1)^{\sum_{l=1}^{[n / 2]} l k_{l}} C_{p}^{k_{0}, k_{1}, \ldots, k_{[n / 2]}} \times \\
& \times \prod_{l=0}^{[n / 2]}\left(C_{n-l}^{l}\right)^{k_{l}} \frac{\left(k_{l}(n-2 l)\right) ! a}{\left(\left(k_{l}(n-2 l)-a+1\right) / 2\right) !\left(\left(k_{l}(n-2 l)+a+1\right) / 2\right) !},
\end{aligned}
$$

где $[n / 2]$ - целая часть числа $n / 2$, а $C_{n-l}^{l}=\frac{(n-l) !}{l !(n-2 l) !}$ и $C_{p}^{k_{0}, k_{1}, \ldots, k_{[n / 2]}}=\frac{p !}{k_{0} ! k_{1} ! \ldots k_{[n / 2]} !}-$ биномиальный и полиномиальный коэффициенты. Для алгебр большего ранга аналогичные формулы нам пока получить не удалось. 


\section{4. ФУНКЦИИ КРАТНОСТЕЙ И ПОЛИНОМЫ ЧЕБЫШЁВА}

Для получения явного вида функции кратностей нам было достаточно свойств сингулярных элементов алгебры g. В этом разделе мы обратимся к свойствам характеров. Известно, что характеры фундаментальных представлений алгебры $s l(n+1)$ являются элементарными симметрическими полиномами в алгебре формальных экспонент:

$$
z_{k}=\operatorname{Ch} L^{\omega_{k}}=\sum_{\nu \in W \circ \omega_{k}} e^{\nu} .
$$

Вейль-инвариантный элемент алгебры формальных экспонент является полиномом по переменной $z_{k}$. В частности, таковым является всякий полином, соответствующий характеру $\mathrm{Ch} L^{\lambda}$ алгебры $\operatorname{sl}(n+1)$, а именно $\mathrm{Ch} L^{\lambda}=U_{\lambda}\left(z_{1}, z_{2}, \ldots, z_{n}\right)$. Здесь $U_{\lambda}$ суть мультивариантные полиномы Чебышёва второго рода [13]. Полученные в предыдущем разделе формулы имеют непосредственное отношение к свойствам полиномов Чебышёва. Прежде всего, рассмотрим эти свойства на примере алгебры $A_{1}$.

4.1. Симметрические полиномы для алгебры $A_{1}$. Пусть $L^{[1]}-$ фундаментальный модуль, $L^{[n]}$ - модуль старшего веса $n \omega$. Перепишем разложение

$$
\left(\mathrm{Ch} L^{[1]}\right)^{p}=\sum_{n=0}^{p} m([n], p) \operatorname{Ch} L^{[n]}
$$

в терминах (унивариантных, т. е. стандартных) полиномов Чебышёва $\mathrm{Ch} L^{[1]}=U_{1}=$ $z, \mathrm{Ch} L^{[n]}=U_{n}(z)$ :

$$
\left(U_{1}(z)\right)^{p}=z^{p}=\sum_{\substack{0 \leqslant n \leqslant p, \sigma(n)=\sigma(p)}} m([n], p) U_{n}(z)
$$

здесь, поскольку четности $\sigma$ чисел $n$ равны четности $\sigma(p)$, коэффициент

$$
m([n], p)=\frac{p !(n+1)}{((p-n) / 2) !((p+n+2) / 2) !}
$$

есть функция кратности модуля $L^{[n]}$ (см. формулу (7)). В такой формулировке функция $m([n], p)$ описывает элементы матрицы перехода между двумя базисами в пространстве симметрических полиномов. Явное выражение для функции кратности означает, что (для $\sigma(n)=\sigma(p)$ ) нам известны все скалярные произведения вида

$$
\left\langle U_{1}^{p}(z), U_{n}(z)\right\rangle=\frac{p !(n+1)}{((p-n) / 2) !((p+n+2) / 2) !} .
$$

4.2. Мультивариантные симметрические полиномы. Формула (6) дает возможность вычислить значение скалярного произведения мультивариантных полиномов Чебышёва $\left\langle U_{1}^{p}\left(z_{1}, \ldots, z_{n}\right), U_{\lambda}\left(z_{1}, \ldots, z_{n}\right)\right\rangle$ (область задания многомерных 
полиномов Чебышёва и выражение для меры интегрирования приведены в работе [13]). Заметим, что функции кратности содержат важную информацию о свойствах симметрических полиномов, поскольку вычисления подобных произведений посредством интегрирования мультивариантных полиномов представляют исключительную сложность.

\section{5. ЗАКЛЮЧЕНИЕ}

Разработан алгоритм подсчета кратностей в разложении $p$-й тензорной степени первого фундаментального модуля на неприводимые подмодули для алгебр серии $A_{n}$, который основан на использовании симметрии Вейля. Построена функция кратности $M_{\left(A_{n}\right)\left\{a_{i}\right\}}^{\bigotimes^{p} \omega}$, т. е. установлена зависимость кратности подмодуля со старшим весом $\mu$ от тензорной степени $p$.

Функция кратности в точках доминантных весов может рассматриваться как подсистема структурных констант алгебры (мультивариантных) полиномов Чебышёва. Мы показали, что удается зафиксировать в явном виде часть алгебры полиномов Чебышёва, связанную со степенями $z_{1}^{p}$. Это означает, что известно разложение мономов $z_{1}^{p}$ по полиномам Чебышёва. В случае алгебры $s l(2)$ значения функции кратности $M_{\left(A_{1}\right)\{a\}}^{\otimes^{p} \omega}$ на решетке доминантных весов суть матричные элементы матрицы перехода от базиса $\left\{z^{p}\right\}$ к базису полиномов Чебышёва.

Предложенный алгоритм применим к тензорным степеням произвольного модуля простой алгебры Ли. Непосредственным результатом такого подхода, основанного на свойствах сингулярных весов, является система конечноразностных уравнений для функции кратности. Далеко не всегда удается построить общее решение такой системы, как это удалось для модуля $L^{\omega_{1}}$ алгебр $A_{n}$. Особую сложность представляют ситуации, когда степень полинома (функции кратности при фиксированных $a_{i}$ ) превышает число ее целочисленных корней. В этом случае для задания функции кратности симметрии Вейля может оказаться недостаточно.

Благодарности. Авторы выражают благодарность организаторам Международного семинара "Классические и квантовые интегрируемые системы" (CQIS-2011) за радушие и гостеприимство. Работа выполнена при поддержке РФФИ (гранты № 09-01-00504, 12-01-00207) и фонда "Династия".

\section{Список литературы}

[1] P. P. Kulish, N. Manojlović, Z. Nagy, J. Math. Phys., 51:4 (2010), 043516, 15 pp., arXiv: 0910.4036 .

[2] S. Kumar, "Tensor product decompositions", Proceedings of the International Congress of Mathematicians (Hyderabad, India, 2010), v. 3: Invited Lectures, ed. R. Bhatia, Hindustan Book Agency, New Delphi, 2010, 1226-1261; Remarks on fermionic formula, arXiv: math/9812022.

[3] A. N. Kirillov, N. Yu. Reshetikhin, J. Math. Sci., 80:3 (1996), 1768-1772.

[4] M. Kleber, Internat. Math. Res. Notices, 1997:4, 187-201, arXiv: q-alg/9611032.

[5] M. Kleber, Finite dimensional representations of quantum affine algebras, arXiv: math/9809087.

[6] V. Chari, Internat. Math. Res. Notices, 2001:12, 629-654, arXiv: math/0006090.

[7] G. Hatayama, A. Kuniba, M. Okado, T. Takagi, Y. Yamada, Remarks on Fermionic formula, arXiv: math/9812022. 
[8] G. Hatayama, A. Kuniba, M. Okado, T. Takagi, Z. Tsuboi, "Paths, crystals and Fermionic formulae", MathPhys Odyssey 2001, Progress in Mathematical Physics, 23, eds. M. Kashiwara, T. Miwa, Birkhäuser, Boston, MA, 2002, 205-272, arXiv: math/0102113.

[9] S. Naito, D. Sagaki, Commun. Math. Phys., 263:3 (2006), 749-787, arXiv: math/0503287.

[10] A.W. Knapp, Lie Groups Beyond an Introduction, Progress in Mathematics, 140, Birkhäuser, Boston, MA, 2002.

[11] М. Ильин, П. Кулиш, В. Ляховский, Алгебра и анализ, 21:2 (2009), 52-70.

[12] A. Klimyk, K. Schmüdgen, Quantum Groups and Their Representations, Texts and Monographs in Physics, Springer, Berlin, 1997.

[13] R. J. Beerends, Trans. Amer. Math. Soc., 328:2 (1991), 779-814. 